\title{
Linx
}

LinX Revue des linguistes de l'université Paris X Nanterre

45 | 2001

Invariants et variables dans les langues. Études typologiques

\section{La typologie et les universaux}

\section{Bernard Pottier}

\section{OpenEdition}

\section{Journals}

Édition électronique

URL : http://journals.openedition.org/linx/717

DOI : $10.4000 /$ linx. 717

ISSN : 2118-9692

Éditeur

Presses universitaires de Paris Nanterre

\section{Édition imprimée}

Date de publication : 1 décembre 2001

Pagination : 19-24

ISSN : 0246-8743

\section{Référence électronique}

Bernard Pottier, "La typologie et les universaux », Linx [En ligne], 45 | 2001, mis en ligne le 18 juin 2012, consulté le 02 mai 2019. URL : http://journals.openedition.org/linx/717 ; DOI : 10.4000/linx.717

Ce document a été généré automatiquement le 2 mai 2019.

Département de Sciences du langage, Université Paris Ouest 


\section{La typologie et les universaux}

\section{Bernard Pottier}

1 1. Depuis une trentaine d'années, on constate une floraison d'études de typologie linguistique, certaines étant organisées sur le plan aréal (ex. EUROTYP, dirigé par Ekkehard König, qui produit neuf gros volumes chez Mouton-de Gruyter ; MEDTYP, dirigé par Paolo Ramat et qui concerne l'ensemble méditerranéen) ou mondial (ex. UNITYP, réalisé par Hansjakob Seiler, dont le huitième volume vient de paraître chez Gunter Narr), sans compter les nombreuses autres études individuelles.

2 Cette abondance de matériaux ne débouche pas toujours sur une réflexion généralisante, de nature hypothétique naturellement, mais nécessaire pour une compréhension des phénomènes fondamentaux sous-jacents à la multiplicité des solutions apportées par chacune des langues. H. Seiler propose de situer sur un axe continu allant d'un plus d' « indicativité » à un plus de " prédicativité » une dizaine de grandes "dimensions » cognitivo-conceptuelles. Nous-même posons un parcours à trois phases (le trimorphe) instancié dans les aires existentielle, spatiale, temporelle, notionnelle et modale (cf. Représentations mentales et catégorisations linguistiques, Peeters, Louvain-Paris, 2000, 318 pages).

3 D’une façon générale, on peut définir ainsi la typologie :

« Etude des types de solutions que les langues apportent à l'expression des événements conceptualisés et modalisés par l'énonciateur. »

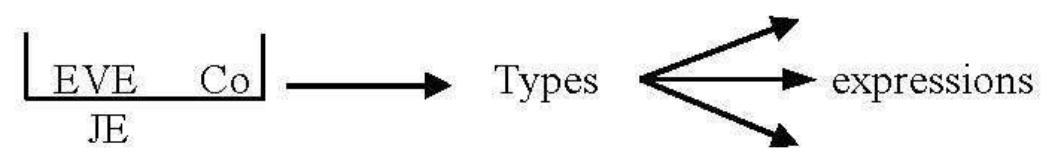

Domaine conceptuel

Domaine des langues

(mental) 

appropriée à la conceptualisation immédiate : dromadaire, Saturne, éparpiller, graisseux, si c'est le cas. S'il y a une volonté de distanciation, on a recours aux opérations META-, et PERI-. Ce phénomène à choix multiple portant sur le lexème est la POLYNOMIE.

7 Parallèlement, le choix de constructions syntaxiques (ou syntaxies) reste à la discrétion de l'énonciateur qui préférera, par exemple, "le Préfet a nommé le directeur » à «le Préfet a procédé à la nomination du directeur» ou «le directeur a été nommé par le Préfet ", etc... On constate ici une POLYSYNTAXIE.

Les deux phénomènes, polynomie et polysyntaxie, constituent les deux réalisations principales (et combinables) de la POLYSEMIOSE, illustrée par le fait que tout élément de catégorisation conceptuelle peut être exprimé à travers de nombreuses solutions morphosyntaxiques (en relation parasynonymique) :

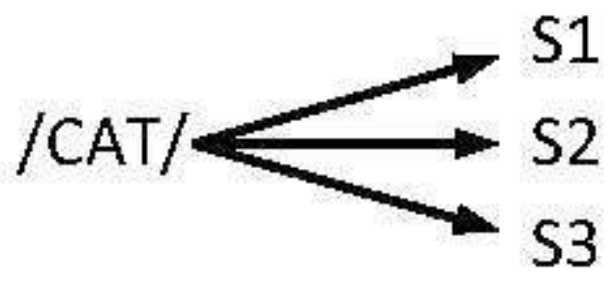

Ex. :

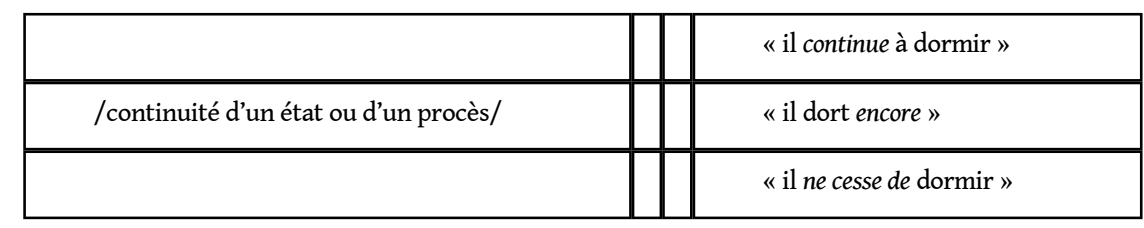

Un graphe, qui trouvera sa place dans un microsystème d'ensemble, pourrait être :

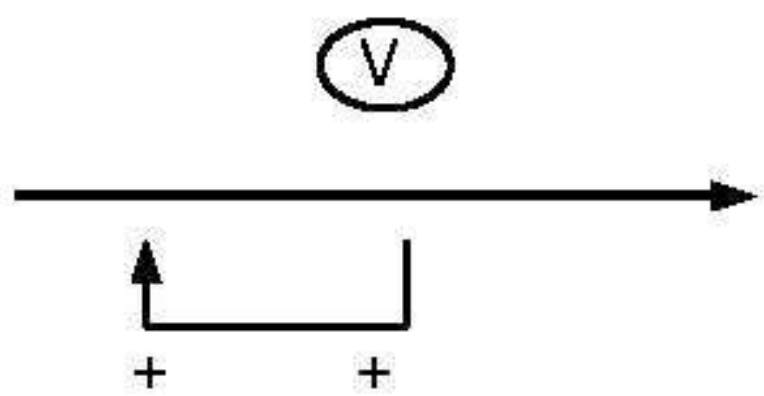

10 Au point de visée (v), le procès existe, et il existait déjà à un point antérieur sur l'axe du temps sans interruption. C'est l'invariant (INV) que l'on trouve sous les trois solutions citées, chacune d'elles ajoutant sa propre spécificité (d'où la parasynonymie). 
11 Dans cette optique, l'étiquette de /virtuel/, proposée par G. Lazard (Papers... to Vl. Nedjalkov, Tübingen, 1998, p. 245), convient pour des solutions linguistiques dites: "prospectif, désidératif, débitif, potentiel, inceptif, éventuel, habituel, subjonctif, optatif » qui, pour nous, ont toutes en commun un noème sous-jacent de type « prospectivité » :

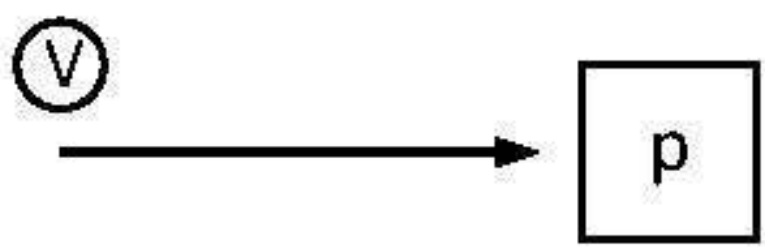
situé en phase 1 du trimorphe ( 2 = inspectivité ou actuel ; 3 = rétrospectivité ou réalisé).

3. Parcourons la totalité du mécanisme onomasiologique :

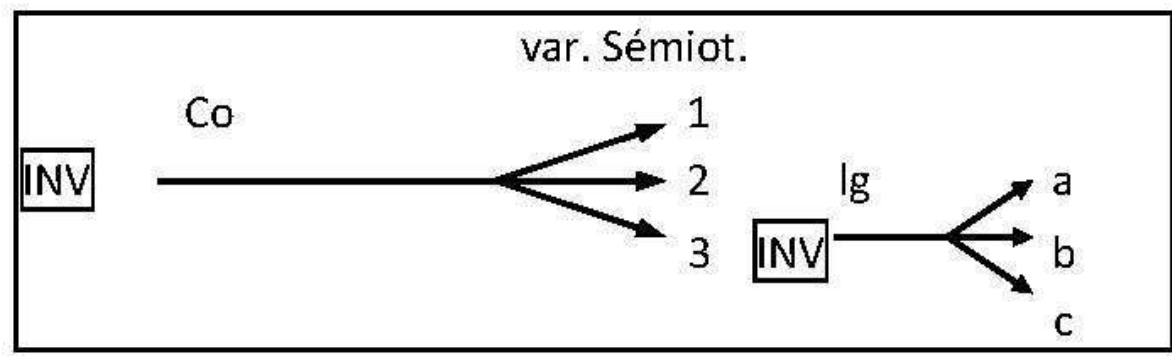

Ex. :

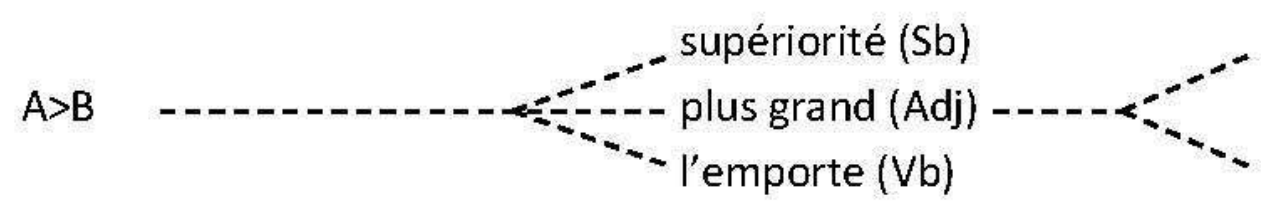

14 La relation $A>B$ est universelle (invariant conceptuel) et toutes les langues l'exprimeront. Elles pourront y attacher une valeur culturelle (la supériorité sera faste dans certains domaines, néfaste dans d'autres, etc...).

Les solutions linguistiques sont toujours multiples :

«Paulus doctior est quam Petrus»

«Paulus doctior est Petro »

16 Le français a des variantes sémiologiques qui entraînent le choix de classes syntaxiques différentes :

« la supériorité de A sur B »

« A est supérieur à $\mathrm{B}$ »

«A l'emporte en x sur B », etc...

17 Et une fois un invariant de langue choisi, l'énonciateur a encore des choix supplémentaires : 

a) « A est supérieur à $\mathrm{B}$ »
b) « B est inférieur à $\mathrm{A}$ »
c) «il y a supériorité de A sur B »
d) « il y a infériorité de B par rapport à A », etc...

On voit ainsi que la considération de la représentation d'un phénomène au niveau mental abstrait permet de comprendre à la fois l'affinité entre ces solutions grammaticalisées (que j'appelle isophasie ou «isosémie au niveau conceptuel»), et les modulations apportées par chaque langue.

4. On doit tenir compte également de la morphosémiologie. De proche en proche, on constate un continuum dans la distension entre la morphologie liée et la morphologie libre :

je parlerai, j'ai à parler, je me dois de parler

je veux parler, je veux bien parler, $j$ 'aurais bien voulu parler

Pour étiqueter une catégorie, le linguiste se fonde, bien souvent, sur la grammaticalisation formelle des langues. Ainsi les grammaires du turc parlent-elles de :

possibilitatif « pouvoir venir »

impossibilitatif «ne pas pouvoir venir

nécessitatif « devoir venir »

Le français pourrait prétendre à un

volitif «P. veut te recevoir»

acceptatif «P. veut bien te recevoir»

ou encore à un

forclusif « un ex-député, un ancien ministre ».

Certaines langues ont

un dotatif « avec sucre, sucré, colourful »

un privatif « sans sucre, colourless"

et le turc ev-li " pourvu d'une maison », " marié », rejoint l'espagnol casado.

En poursuivant l'analyse, on peut distinguer deux sous-types de DOTATIF :

\begin{tabular}{|l||l||l|}
\hline /qui possède par nature/ & & /qui possède par accident/ \\
\hline \hline « un chemin boueux » & & «des chaussures boueuses » \\
\hline esp. «es lodoso» & & «tiene lodo » « está con lodo » \\
\hline
\end{tabular}

et deux types de PRIVATIF :

\begin{tabular}{|l||l|l|}
\hline /qui n'a jamais eu/ & & /qui n'a plus/ \\
\hline «je suis sans voiture » & & « je suis sans voiture » \\
\hline (je ne sais pas conduire) & & (elle est en panne) \\
\hline
\end{tabular}

23 Ces dénominations ad-hoc prennent leur justification lorsqu'on se réfère à un microsystème qui amorce la présentation en trois phases que nous proposons plus loin. En voici un exemple :

Une « chrono-logie » s'instaure : 


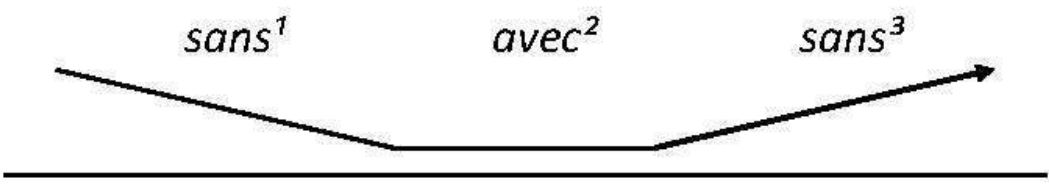

\section{AVANT d'avoir \\ avoir \\ APRES avoir eu} mécanisme cinétique à trois phases :

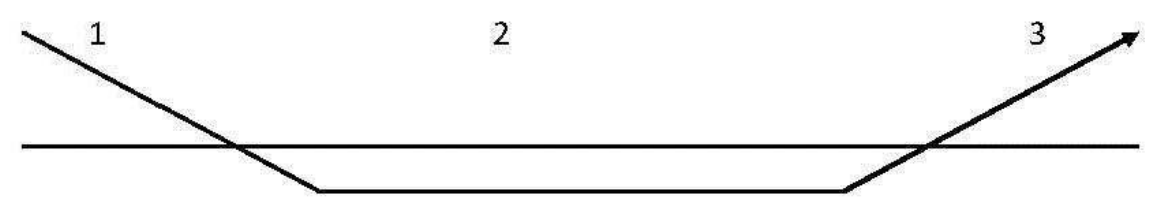

in-ermis

arm-atus

de-arm-atus

im-berbis

barb-atus

vêtu (é-barb-é)

dé-vêtu

mis à nu

dé-nudé

NB. de- est privatif dans « dé-vêtu » et intensif dans « dé-nudé », continuant ainsi les « têtes de Janus » du latin liées au préfixe de-.

5. Cette même démarche va se retrouver dans des domaines apparemment très éloignés si l'on s'en tient aux chapitres traditionnels des grammaires.

Que l'on compare :

\begin{tabular}{|l|l|l|}
\hline «je veux un carnet rouge » & « je veux ce carnet rouge » & « je veux le carnet rouge » \\
\hline \hline 1 & 2 & 3 \\
\hline
\end{tabular}

En 1, il y a mouvement prospectif vers une entité qui est en vue dans l'esprit.

En 2, il y a contact (visuel ou mémoriel) avec l'objet.

En 3, l'énoncé nécessite un complément d'information car il se réfère à du déjà vu, déjà connu, et donc signifie "je veux le carnet rouge que tu as chez toi », ou à tout autre cotexte.

On peut donc représenter ainsi le trimorphe. 


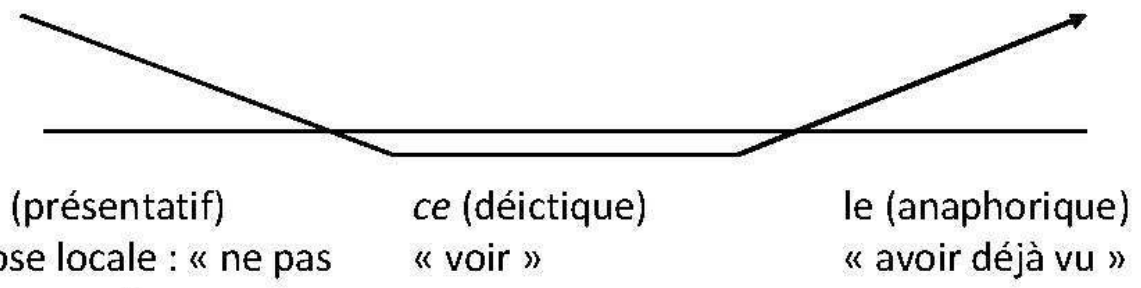

encore voir »

27 6. Autre exemple, dans le domaine de la modalité épistémique. Le SAVOIR, qui exprime un contact entre l'énonciateur et le propos, possède un AVANT (le parcours qui mène au SAVOIR, donc une incertitude) et un APRES (qui s'éloigne du SAVOIR de l'énonciateur, lequel "prend ses distances » parce qu'une médiation s'est produite liée, par ex., à une intervention extérieure).

On peut figurer ce mécanisme ainsi, en fonction de l'engagement du JE énonciateur :

\begin{tabular}{|l|l|l|}
\hline 1 & 2 & 3 \\
\hline & SAVOIR & \\
\hline distance d'AVANT & & distance d'APRES \\
\hline \hline «je crois qu'il a divorcé » & «je sais qu'il a divorcé » & «il aurait, dit-on, divorcé » \\
\hline $\begin{array}{l}\text { (je ne le vois plus avec sa } \\
\text { femme) }\end{array}$ & (j'ai vu l'acte notarié) & $\begin{array}{l}\text { (la rumeur me le fait } \\
\text { croire) }\end{array}$ \\
\hline $\begin{array}{l}\text { élaboration personnelle et } \\
\text { responsable du JE }\end{array}$ & $\begin{array}{l}\text { le JE n'est plus responsable, cela } \\
\text { est « évident » }\end{array}$ & $\begin{array}{l}\text { le JE devient dépendant } \\
\text { des AUTRES }\end{array}$ \\
\hline
\end{tabular}

Ceci constitue un microsystème possible, étant donné la morphosémiologie assez nette du français à ce sujet.

7. Un autre avantage de cette représentation triphasée est qu'un schème noémique, ou noémie, permet de mettre en valeur les métaphores liées à une même phase : 


$\begin{array}{ll}\text { ouvrir } & \\ \text { allumer } & \text { fermer } \\ \text { écrire } & \text { éteindre } \\ \text { exciter } & \text { effacer } \\ \text { partir } & \text { apaiser } \\ & \text { s'arrêter }\end{array}$

Penser à :

italien : fermare « arrêter»

portugais : apagar « effacer, « éteindre »

Ces parcours événementiels font référence soit à l'existence, soit à l'espace, soit à la modalité, avec des transferts toujours théoriquement possibles :

apaiser la soif = la faire disparaître

allumer des passions = faire naître

\section{Conclusions}

32 L'étude de nature typologique permet de dégager des traits ou comportements communs à plusieurs langues (lesquels conduiront à établir des différences plus ou moins importantes) (GENERALITÉ).

Un premier niveau d'abstraction amène à poser des notions qui, organisées, peuvent jouer le rôle de tertium comparationis (COMPARABILITÉ).

Un second niveau, qui nous paraît indispensable et qui légitimement relève de la compétence du linguiste, consiste à construire une hypothèse sur les mécanismes mentaux simples et itératifs qui sous-tendent les catégorisations des objets et des représentations du Monde. Ce sont les modèles trimorphiques que nous proposons dans notre ouvrage cité au début de cet article (UNIVERSALITE).

\section{AUTEUR}

\section{BERNARD POTTIER}

